Short Communication

\title{
Multifunctional profiling of triple-negative breast cancer patient-derived tumoroids for disease modeling
}

\author{
Evan F Cromwell ${ }^{\mathrm{a}, *}$, Oksana Sirenko ${ }^{\mathrm{b}}$, Ekaterina Nikolov ${ }^{\mathrm{a}}$, Matthew Hammer ${ }^{\mathrm{b}}$, \\ Courtney K Brock ${ }^{c}$, Margarite D Matossian ${ }^{c}$, Madlin S Alzoubi ${ }^{c}$, Bridgette M Collins-Burow ${ }^{c}$, \\ Matthew E Burow ${ }^{\mathrm{c}}$ \\ a Protein Fluidics, Inc., USA \\ ${ }^{\mathrm{b}}$ Molecular Devices, LLC, USA \\ c Tulane University School of Medicine, USA
}

\section{A R T I C L E I N F O}

\section{Keywords:}

Microfluidics

Tumoroids

High-content Imaging

Metabolism

Patient-derived

Disease modeling

Breast cancer

\begin{abstract}
A B S T R A C T
3D cell models derived from patient tumors are highly translational tools that can recapitulate the complex genetic and molecular compositions of solid cancers and accelerate identification of drug targets and drug testing. However, the complexity of performing assays with such models remains a hurdle for their wider adoption. In the present study, we describe methods for processing and multi-functional profiling of tumoroid samples to test compound effects using a novel flowchip system in combination with high content imaging and metabolite analysis. Tumoroids were formed from primary cells isolated from a patient-derived tumor explant, TU-BcX-4IC, that represents metaplastic breast cancer with a triple-negative breast cancer subtype. Assays were performed in a microfluidics-based device (Pu·MA System) that allows automated exchange of media and treatments of tumoroids in a tissue culture incubator environment. Multi-functional assay profiling was performed on tumoroids treated with anti-cancer drugs. High-content imaging was used to evaluate drug effects on cell viability and expression of E-cadherin and CD44. Lactate secretion was used to measure tumoroid metabolism as a function of time and drug concentration. Observed responses included loss of cell viability, decrease in E-cadherin expression, and increase of lactate production. Importantly, the tumoroids were sensitive to romidepsin and trametinib, while showed significantly reduced sensitivity to paclitaxel and cytarabine, consistent with the primary tumor response. These methods for multi-parametric profiling of drug effects in patient-derived tumoroids provide an in depth understanding of drug sensitivity of individual tumor types, with important implications for the future development of personalized medicine.
\end{abstract}

\section{Introduction}

The use of patient-derived tumor tissues has transformed the field of drug and target discovery research, providing a translational tool and physiologically relevant system to evaluate tumor biology [1,2]. This is important as only approximately $7 \%$ of oncology drugs make it through clinical trials [3]. Furthermore, tumor heterogeneity and resistance to available drugs present a great unmet need for development of new therapeutics. There has been a significant transition from traditional 2D assays to more complex 3D cell model systems [4-6]. 3D models are believed to more accurately recapitulate the cellular compositions of human in vivo environment and serve as a better predictive tool for drug discovery $[7,8]$. An example of this method is the use of patient-derived organoids (PDO) for oncology research $[2,9,10]$. Patient-derived tumors reflect the heterogeneity of patient tumor tissues and enrich for the pres- ence of self-renewing cancer stem cells (CSC) that can be expanded over multiple passages to produce large numbers of tumoroids (derived from isolated cells) or organoids (derived from digested tumors), that maintain molecular characteristics of the original tumor.

While significant progress has been made in development of more advanced cell models to recapitulate the in vivo environment, there are still hurdles for performing complex assays with those samples. Organoids are derived from small volume of tissues, have limited ability to expand, are fragile, and can be subject to loss or damage from technical aspects of organoid processing. This is especially important for pipetting steps including media exchange, supernatant sampling, or immunofluorescence staining. Optimization of these processes is critical for successful assays with complex tumor micro-tissues. Furthermore, to accurately assess drug response in vitro and to predict outcomes in biological systems, it is crucial to assess multiple parameters and responses of such

\footnotetext{
* Corresponding author.

E-mail address: ecromwell@proteinfluidics.com (E.F. Cromwell).
} 
A

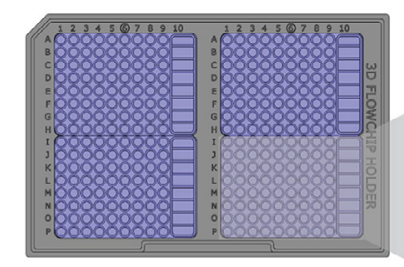

$\mathrm{D}$

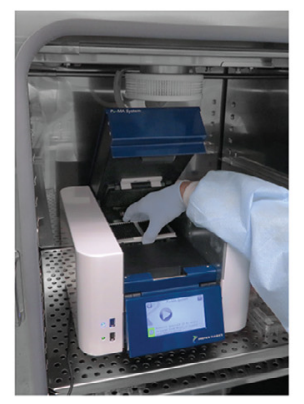

$\mathrm{B}$

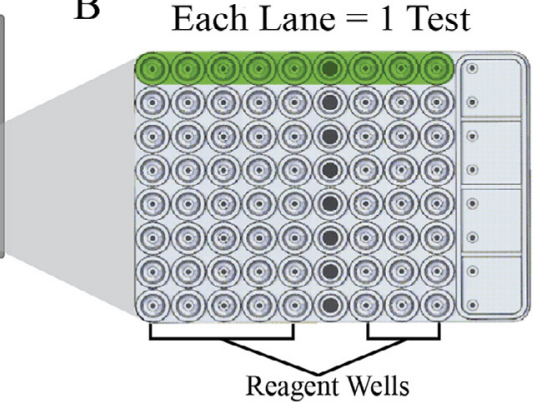

Well Connections Layout

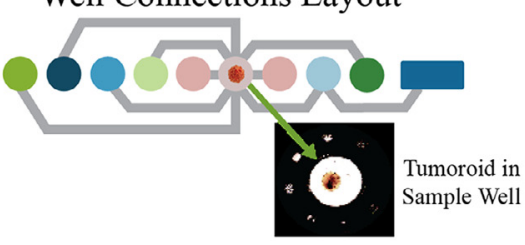

$\mathrm{C}$

\section{Sample Well}

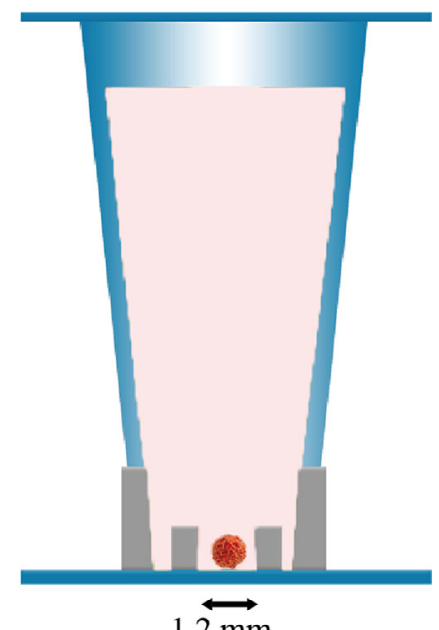

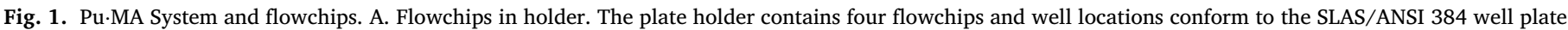

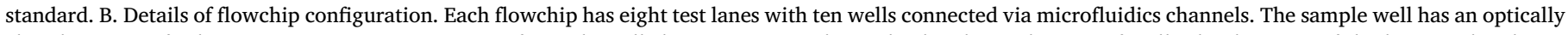

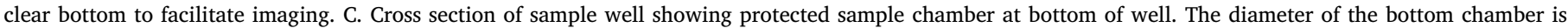

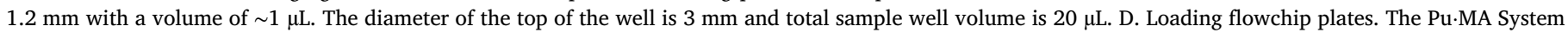
fits into standard size incubators. The plates are loaded using a clam-shell operation. This quickly interfaces the pneumatic manifold gasket to all 320 wells.

translational models. This can be done using larger numbers of tumoroids with single data points per sample or apply a more elegant and efficient way by collecting multiplexed data from individual tumoroids using multifunctional profiling (MFP).

To facilitate assessment of drug treatment effects in tumoroids we used a microfluidic device, the Pu.MA System and flowchips, for semiautomated tumoroid assays in combination with high content imaging (Fig. 1) [11]. The Pu.MA System enables liquid transfers in special labware (flowchips) by applying selective pneumatic pressure. The flowchip is designed with sample wells connected to multiple adjacent wells (Fig. 1B) that can accommodate various assay reagents allowing media exchange, sample staining, wash steps, and other processing to be performed without disruption to, or loss of, the 3D sample. The bottom of the sample chamber is thin, optically clear plastic compatible with high resolution fluorescence imaging. This novel assay method using microfluidics enables automation of 3D cell-based cultures that mimic in vivo conditions, performs multi-dosing protocols and multiple media exchanges, provides gentle and convenient handling of tumoroids and organoids, and allows a wide range of assay detection modalities. In this paper we extend use of supernatant sampling shown previously for a single end-point [11] to a multi-point time-course of metabolite secretion that allows dynamic profiling of tumor cell metabolism response to therapeutic compounds. We also show utility for immunofluorescence staining to provide biomarker characterizations.

We present here results for breast cancer disease modeling using tumoroids formed from primary cells isolated from a patient-derived tumor, TU-BcX-4IC [12,13]. This particular cell-based model was chosen in the present work as an example to show the capabilities of the assay methods described here. TU-BcX-4IC represents a rare breast cancer subtype, metaplastic breast cancer (MBC), and is classified as a triple negative breast cancer (TNBC) pathologic subtype with diverse histologic features including epithelial and mesenchymal cellular composition $[13,14]$. We selected this patient-derived model because it represents an example of a highly heterogeneous phenotype of breast cancer. TNBC tumors have an aggressive clinical presentation due to high rates of metastasis, recurrence and chemoresistance. The original patient's tumor for the TU-BcX-4IC model exhibited rapid pre-operative growth despite conventional combination therapy with adriamycin (doxorubicin), cyclophosphamide and paclitaxel. In this study we assessed the effects of the targeted inhibitors romidepsin (primarily targets HDAC1/2) and trametinib (MEK1/2 inhibitor), as well as conventional systemic chemotherapeutics paclitaxel and cytarabine on cell viability and phenotypic change of primary derived microtissues. Importantly, resistance of tumoroids to chemotherapeutic agents observed in our experiments were consistent with the resistant tumor response observed within the patient [12], emphasizing the translational utility of our novel technique. This work demonstrates the improved workflow of the assay with microfluidics-based Pu.MA System for in vitro drug discovery research in testing structurally delicate 3D cell models which can be applied to all solid tumor types.

\section{Materials and methods}

\section{PDX methods, cell lines and tumoroid formations}

The methods for generating tumoroids and PDX organoids (PDX-O) have been previously described $[2,12]$. The tumor sample from TU-BcX4IC (also hereafter denoted as '4IC') implanted into SCID/Beige mice exhibited rapid tumor growth, with 14 days to reach maximal tumor volume $>1000 \mathrm{~mm}^{3}$, compared to the mean length of time to maximum volume of other established TNBC PDX models [15]. Immunohistochemistry staining of 4IC revealed both epithelial and mesenchymal histologies, and high cellularity, which was consistent throughout all serial transplants. Tumoroids were formed from cells that were isolated from the 4IC tumor and were considered primary cells (Fig. 2). Single cell suspensions containing 2000 cells per tumoroid were plated in lowattachment dishes and incubated with $5 \% \mathrm{CO}_{2}$ and $37{ }^{\circ} \mathrm{C}$ conditions for $48 \mathrm{~h}$ until they formed tight tumoroids. 4IC cells were cultured with Advanced DMEM supplemented with glucose, NEAA, $2 \mathrm{mM}$ glutamine and insulin $120 \mu \mathrm{g} / \mathrm{L}, 10 \%$ FBS (Gibco 12491-015). For metabolic assays, tumoroids were cultured with DMEM $+10 \%$ dialyzed serum $(2 \mathrm{mM}$ glutamine, $5 \mathrm{mM}$ glucose, without phenol red). Tumoroids were formed using either single well low attachment plates (ULA 384, Corning) or low attachment multicavity inserts (AggreWell 400, STEMCELL Technologies). Spheres formed by both methods exhibited high uniformity consistent with that observed with other cell models formed in 384- 
A î
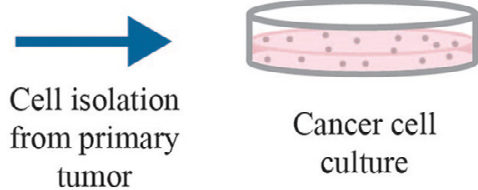
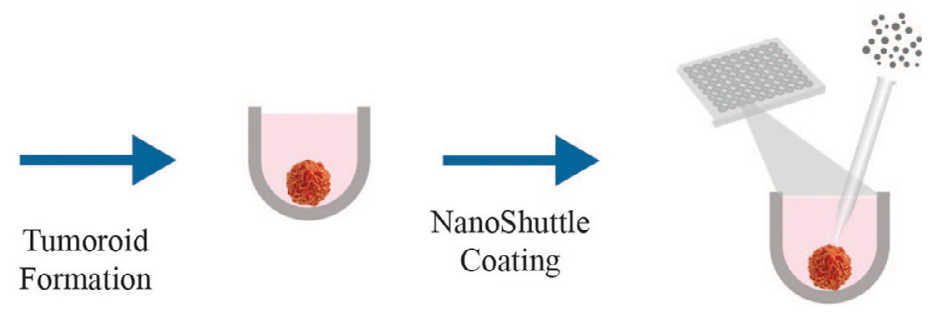

B

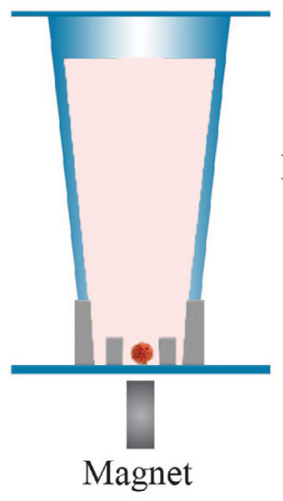

C
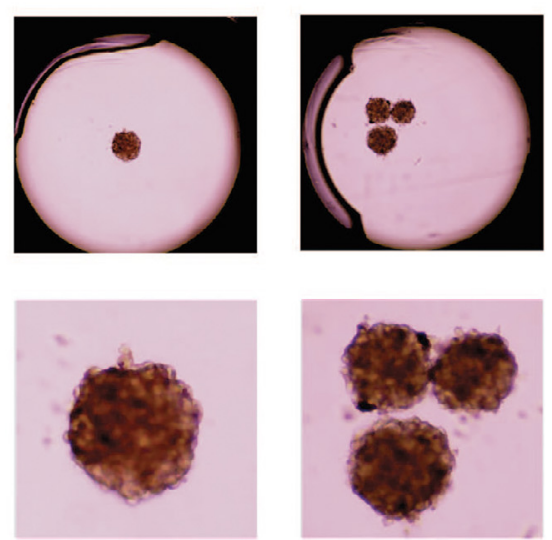

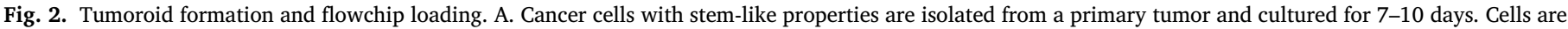

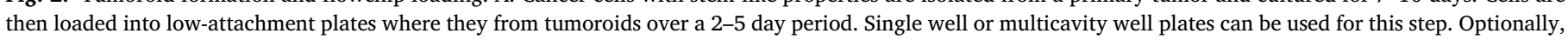

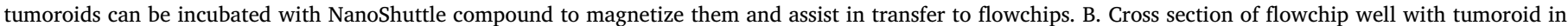

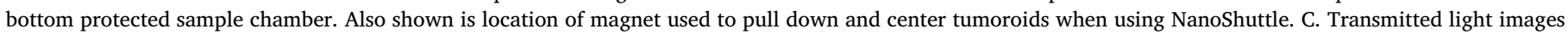

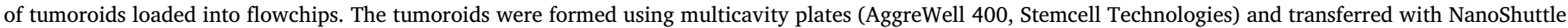
which helped center them in the well.

well low attachment plates [16]. PDX-Os were organoids derived from patient-derived xenograft tissue generated from serial transplantation of TU-BcX-4IC intact tumor pieces in immunocompromised mice. PDX-Os were formed from cells from digested PDX tissue harvested from mice, and then 2000 cells per well were plated in a low attachment 24 well plate and incubated for 7-14 days. PDX-Os were passaged and samples were designated by passage number.

\section{Microfluidic automation}

The Pu-MA System is a low to medium throughput bench-top instrument that operates inside a tissue culture incubator to perform assay protocol steps with organoids, tumoroids, and other 3D cellular structures (Fig. 1). It uses a microprocessor controlled pneumatic system to move fluid between wells via pressure differentials [11]. A key component of the automation system is the Pu.MA System flowchip. The flowchips contain reagents and samples wells that are connected by microfluidic channels. A set of four flowchips are held in a convenient frame that conforms to the SLAS/ANSI 384 well plate standard and enables 32 samples to be tested in parallel. There is a protective chamber for micro-tissue inside the sample wells that prevents disturbance by liquid flow. Assay reagent wells are connected to the sample well via side ports allowing fluid exchange to occur without disturbing micro-tissues in the protected chamber (Fig. 1C). The flowchips are made from black cyclic olefin copolymer (COC) with a thin, optically clear COC bottom suitable for multiple assay read-outs including high resolution imaging.

Micro-tissues, media, compounds, or other reagents are pre-loaded into the flowchip by pipetting, similarly as into a regular microplate. Tumoroids were positioned into a special protected chamber at the bottom of the sample well. In some experiments, tumoroids were coated with magnetic nanoparticles (NanoShuttle, Greiner Bio One) and then positioned in the bottom of the flowchip wells using small magnets
(Fig. 2B) [11]. Tumoroids were incubated with $\sim 0.1 \mu \mathrm{L}$ of NanoShuttle per tumoroid for $2 \mathrm{~h}$ at $37^{\circ} \mathrm{C}$. This helped to center the tumoroids and facilitated imaging. After sample loading, the plate was placed into the $\mathrm{Pu} \cdot \mathrm{MA}$ System located in an incubator $\left(37^{\circ} \mathrm{C}\right.$ and $5 \% \mathrm{CO}_{2}$ ) (Fig. 1D) and reagent exchanges were done automatically through the microfluidic channels using pre-loaded automation protocols. Multiple reagent exchanges were performed sequentially, enabling complex assay protocols to be run in an automated workflow with no manual processing steps. Typically, 8-point concentration response of two compounds were run in duplicates per plate ( 32 total samples). For the lactate secretion measurements, 4-point concentrations were run of three compounds in two to three replicates per plate.

\section{Compound treatments}

For compound screening, chemicals were prepared as $10-100 \mathrm{mM}$ stock solutions in tissue culture-grade DMSO (Sigma-Aldrich, St. Louis, MO). Then compounds were diluted to appropriate concentrations in the culture media. The final concentration of DMSO in media was $0.1 \%$. For the assays, compounds (Sigma-Aldrich) were typically tested in duplicates in a six-point dilution series. Tumoroids were transferred into flowchips and treated by performing an automated media exchange. Cells were then exposed to the various concentrations of compounds for 24 or $48 \mathrm{~h}$.

\section{Viability and immunofluorescence staining}

The method for imaging and high content analysis of 3D tumoroids was previously described [16,17]. Briefly, following incubation with test compounds, tumoroids were stained for $1 \mathrm{~h}$ with a mixture of three dyes: $1 \mu \mathrm{M}$ calcein AM, $3 \mu \mathrm{M}$ of EthD-1, and $33 \mu \mathrm{M}$ Hoechst 33342 (Thermo Fisher, Carlsbad, CA). Dyes were prepared in sterile phosphate buffered 


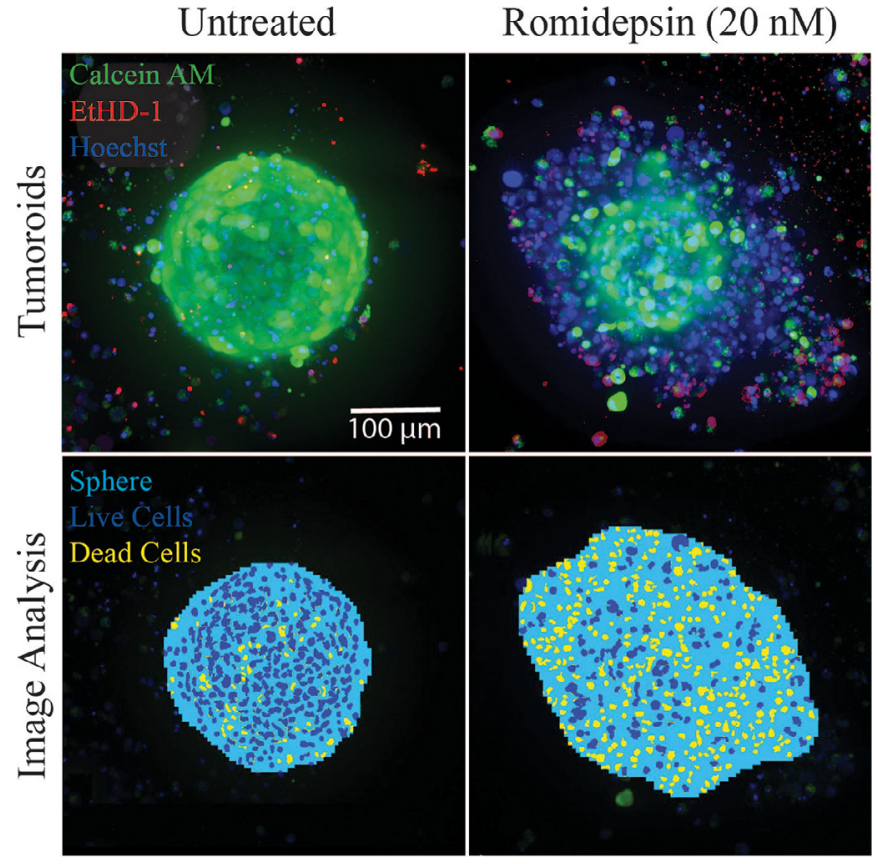

Fig. 3. Phenotypic analysis of tumoroid samples treated with romidepsin by high content imaging. Tumoroid samples were treated with $10 \mathrm{nM}$ of romidepsin for $48 \mathrm{~h}$, then stained live with Hoechst, calcein AM and EthD-1 for $1 \mathrm{~h}$ and imaged using IXM-C instrument with 10x objective. A Z-stack of 30 confocal images of tumoroids were collected $10 \mu \mathrm{m}$ apart, maximum projection confocal images are presented for untreated and treated tumoroids. Nuclei presented in blue, calcein $\mathrm{AM}$ in green, and EthD-1 in red. Lower panel represent image analysis done using custom module editor of MetaXpress software. Analysis marked tumoroid object (blue), live cells (dark blue), dead cells (in yellow).

saline (PBS, Corning). Tumoroids were stained in a Pu·MA System with viability and cell surface biomarkers and imaged on ImageXpress Micro Confocal automated imaging system. Images were analyzed for percent of marker positive cells. For E-cadherin and CD44 detection tumoroids were first fixed with $4 \%$ formaldehyde solution (Sigma) for $30 \mathrm{~min}$, then washed with PBS and stained with directly conjugated FITC mouse antiE-cadherin (p/n 612130, BD Biosciences) and PE anti-human CD44 (p/n 338807, Biolegend) antibodies overnight at 1:100 dilution in the presence of $10 \%$ of Fetal Bovine Serum, then washed and imaged.

\section{Imaging and analysis}

Images of tumoroids were acquired using confocal automatic imaging system, ImageXpress Micro Confocal (Molecular Devices, San Jose, CA), as previously described $[16,17]$ with a 10X Plan Fluor or 20X Plan Apo objectives (Fig. 3). DAPI, FITC and Texas Red filter sets were used for imaging. To image 3D organoids typically a stack of 10-15 images separated by 7-15 $\mu \mathrm{m}$ was acquired, starting at the well bottom and covering approximately the lower half of each tumoroid. Typically, a Zstack of images covered $100-200 \mu \mathrm{m}$ of height for each tumoroid. Image analysis was performed either in 3D using individual Z-stack images, or in $2 \mathrm{D}$ using the $2 \mathrm{D}$ Projection (maximum projection) images of confocal image stacks. Transmitted light images were used for cell culture monitoring or protocol optimization.

Images were analyzed using MetaXpress High-Content Image Acquisition and Analysis Software (Molecular Devices). Count Nuclei or and Cell Scoring application modules were used for nuclear count and live/dead assessment, respectively. A customized analysis for multiparametric measurements was done using a Custom Module Editor (CME). The custom module analysis first identified the tumoroid object using Hoechst staining. Then, individual cells were counted withing tumor- oid objects by nuclear stain and viable cells were identified by presence of calcein AM or by the absence of EthD-1 signal, and dead cells were identified by presence of EthD-1 signal. In some experiments, apoptotic cells were defined using NucView 488 stain (Biotium). Measurements included counts of calcein AM positive or EthD-1 positive cells, tumoroid width, tumoroid area, volume (in 3D analysis), average intensities for calcein AM or EthD-1, counts of all nuclei, and evaluation of average nuclear size and average intensities. $\mathrm{IC}_{50}$ values were determined using 4-parameter curve fit from SoftMax® Pro 6 software (Molecular Devices) or Prism (GraphPad Software).

\section{Lactate secretion assay}

The metabolic response of tumoroids to treatment was determined by measuring lactate secretion in supernatants that were collected from treated and untreated 4IC tumoroids at various timepoints of drug treatment using the Pu.MA System. Supernatant collection was done in the flowchip for each treatment condition in the following way: media with drug was transferred from an adjacent reagent well of the flowchip to the sample well with tumoroid and incubated for $3 \mathrm{~h}$ (Supplementary Fig. 1). After incubation, the media containing secreted lactate was transferred back to the reagent well it came from and replaced with fresh media with drugs from another well for the next $3 \mathrm{~h}$ treatment. The cycle repeated 5 times and resulted in collection of 5 supernatant samples. The first cycle was done with medium only (baseline secretion) followed by 4 treatment cycles for the total treatment duration of $12 \mathrm{~h}$. This approach allows dynamic monitoring of lactate secretion over the course of treatment.

The collected supernatants were stored in the flowchips until the end of the $\mathrm{Pu} \cdot \mathrm{MA}$ protocol then were collected and stored at $-20{ }^{\circ} \mathrm{C}$ until further processing. The supernatant samples were analyzed for lactate levels using luminescence Lactate-Glo assay (Promega). Lactate detection reagent was prepared according to the manufacturer's protocol. Supernatant samples were diluted $1: 400$ in PBS. $10 \mu \mathrm{L}$ of the diluted supernatant was transferred to a solid white 384-well assay plate and $10 \mu \mathrm{L}$ of lactate detection reagent was added to each well. Plates were incubated for $60 \mathrm{~min}$ at room temperature. Luminescence was measured using GloMax plate reader (Promega). Each sample was measured in duplicate. Good luminescence signal levels and signal-to-noise ratios were achieved for this assay from single tumoroid samples (see Supplementary Table 1 and Supplementary Fig. 2). The statistical significance of comparison between groups was determined using one-way or two-way ANOVA. Post hoc tests were run to confirm where the differences occurred between groups. Differences with $p<0.05$ were considered statistically significant.

\section{Luminescence viability assays}

Two luminescence-based viability assays were used in conjunction with the metabolite assays: RealTime-Glo MT [18] (RT-Glo, Promega) and CellTiter-Glo 3D (CTG, Promega). All incubations and luminescence measurements for these assays were done within the flowchips. RT-Glo assay was performed prior to the treatment initiation to assess tumoroid size and viability. RT-Glo reagent was prepared in culture media according to the manufacturer's protocol. $20 \mu \mathrm{L}$ of the prepared RT-Glo solution was added directly to the sample well in the flowchips containing the tumoroid. After that flowchips were incubated for $2 \mathrm{~h}$ at $37{ }^{\circ} \mathrm{C}$. Luminescence signal was measured in a plate reader (GloMax, Promega). CTG 3D assay was done at the end of the incubation of tumoroids with compounds. Flowchips and CTG 3D reagent were equilibrated to room temperature. After that $10 \mu \mathrm{L}$ of media was removed from the sample well with tumoroid and replaced with $10 \mu \mathrm{L}$ of the CTG 3D assay reagent. The flowchip plate was incubated for $40 \mathrm{~min}$ at room temperature and then luminescence signal was measured in a plate reader. 


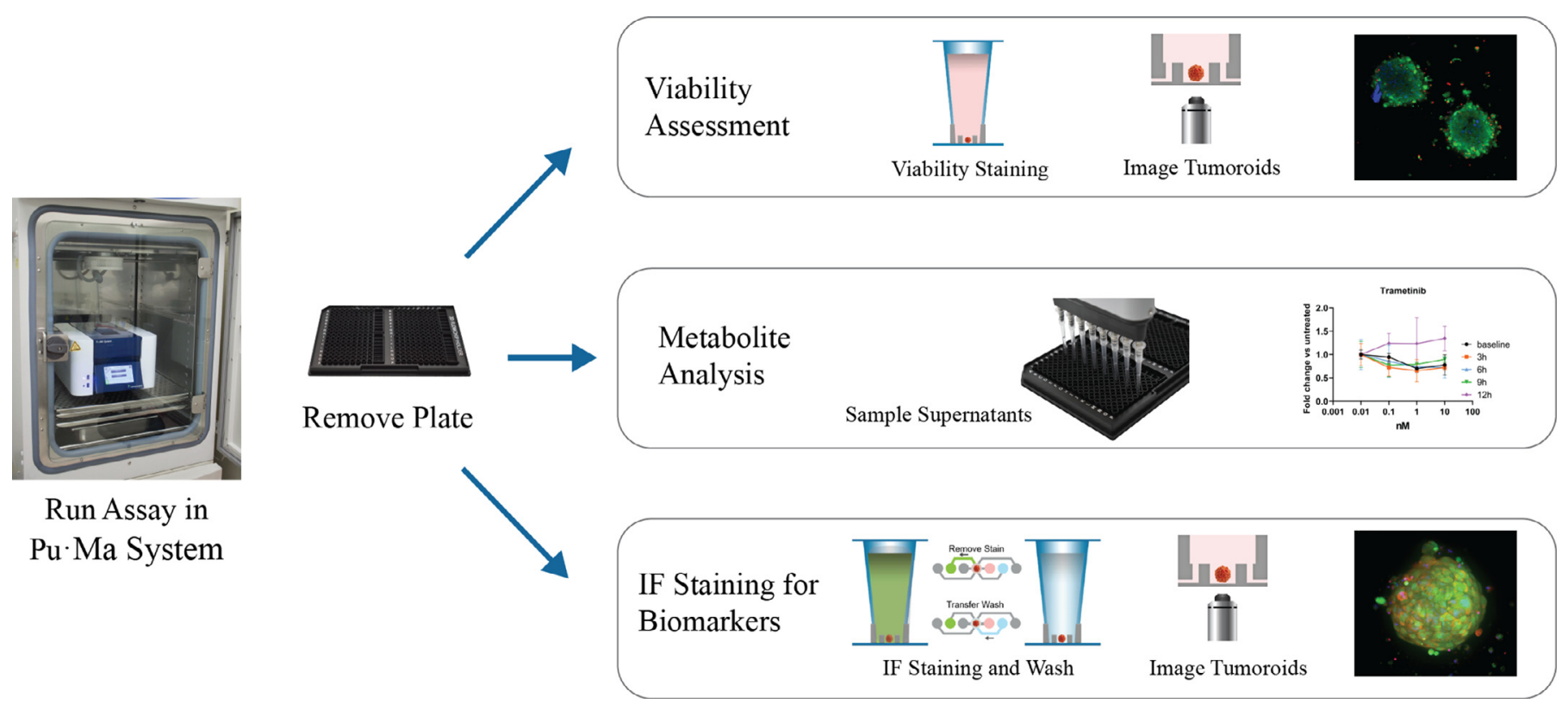

Fig. 4. Multifunctional profiling workflow. Tumoroids are treated in Pu-MA System using automated protocols. Once treatment is finished tumoroids can be stained for viability markers and then imaged and analyzed for live or dead cells. In parallel, supernatant samples can be removed and analyzed for metabolites such as lactate and glutamate, or other secreted factors. Finally, tumoroids can be fixed and stained with antibodies against biomarkers such as E-cadherin and CD44.

\section{Results}

\section{Multifunctional profiling}

In the present study we used a method for assay automation to test compound effects on primary tumor microtissues using automated liquid transfer and high content imaging [11]. Tumoroids were formed from primary cells isolated from a patient-derived tumor sample, TUBcX-4IC (4IC), as described in the Methods section. This cell line has been previously characterized and is used as an example to show capabilities of the methods $[12,13]$. We used two variations of patientderived 3D models: (1) 4IC tumoroids formed from an expanded primary cell line formed in low attachment plates and (2) organoids formed from tissue fragments of mouse xenografts (PDX-O).

Tumoroids were incubated with either media + vehicle (untreated control) or media + compound for 24 to $48 \mathrm{~h}$ to measure phenotypic responses. Both types of microtissues were treated with the following compounds:

- Romidepsin - A histone deacetylase (HDAC) inhibitor.

- Trametinib - Selective reversible allosteric inhibitor of MEK1 and MEK2 activity.

- Paclitaxel - A mitotic inhibitor that interferes with microtubule growth.

In addition, TU-BcX-4IC spheres were tested using the following compound:

\section{- Cytarabine - A DNA polymerase inhibitor.}

The use of the Pu.MA System microfluidics allowed automated sample processing, and enabled multiplex time point sampling of tumoroid secretions which provided multiple end-point readouts (see Fig. 4). These readouts included viability assessment by high content imaging with live staining for calcein AM and EthD-1, changes in shape (size, volume, density, etc), as well as expression of specific biomarkers including E-cadherin and CD44. Media was collected at various time points during treatment to measure secretion of lactate. Additional factors such as cytokines, chemokines, and growth factors can also be measured, but were not part of present study [11]. The small volume of the sample well gives a more concentrated analyte solution and a natural enhancement of sensitivity.

\section{Tumoroid viability response to compounds}

4IC tumoroids were analyzed to assess their response to paclitaxel, romidepsin, trametinib, and cytarabine. Tumoroids were formed in low attachment plates for $48 \mathrm{~h}$ and incubated with compounds for either 24 or $48 \mathrm{~h}$. After treatment and staining, tumoroids were imaged using ImageXpress Micro Confocal Imaging System for various cell viability markers (as described in the Methods section). Confocal imaging provided 3D cellular resolution of tumoroid structures to observe the size and integrity of tumoroids, including the number of total, viable and effected cells. Detailed image analysis allowed determination of phenotypic changes and the impact of compound treatment by the characterization of tumoroids shape, cell count, and viability. Tumoroids were imaged at appropriate wavelengths with a 10X objective; a Z-stack of images was taken using 7-15 $\mu \mathrm{m}$ interval. Only a single site was required with a 10X objective due to the small clear aperture of the flowchip $(\sim 1.2 \mathrm{~mm})$ reducing both acquisition time and image processing time. Images of treated and untreated tumoroids are shown in Fig. 5.

Image analysis was done either in 3D with Z-stack images or in 2D using maximum intensity projection images to identify nuclei, calcein AM positive and EthD-1 positive cells. Cell viability was measured by quantifying percentages of total cells (count nuclei), live cells (EthD1 negative cells, or calcein AM positive), and dead cells (EthD-1 positive or calcein AM negative). Examples of image analysis results from maximum projection images for tumoroids treated with compounds are shown in Fig. 3.

There were marked phenotypic changes observed in the tumoroids after compound treatment: decrease of live cell staining (calcein AM positive) and increase in the number of dead cells (EthD-1 positive). In addition, tumoroids exposed to compounds become partially disintegrated, whereas untreated tumoroids maintained their shape throughout the experiment (Fig. 5A). Phenotypic changes were quantitated by counting numbers of cells positive or negative for different markers and by measuring average intensities of stains, cell areas, or tumoroid areas (Fig. 5B). IC $_{50}$ values for different compound effects were calculated from using 4-parametric curve fits. All tested compounds, except 
A

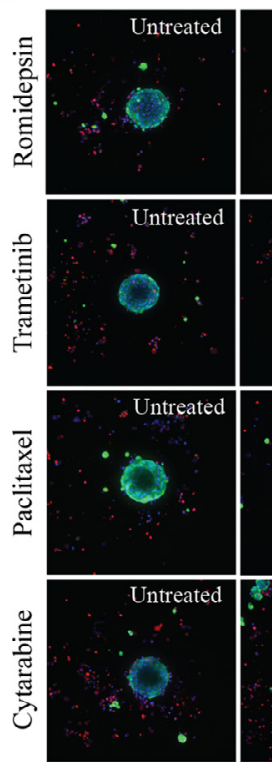

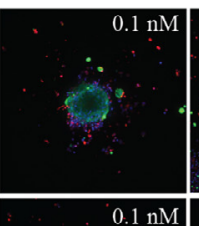
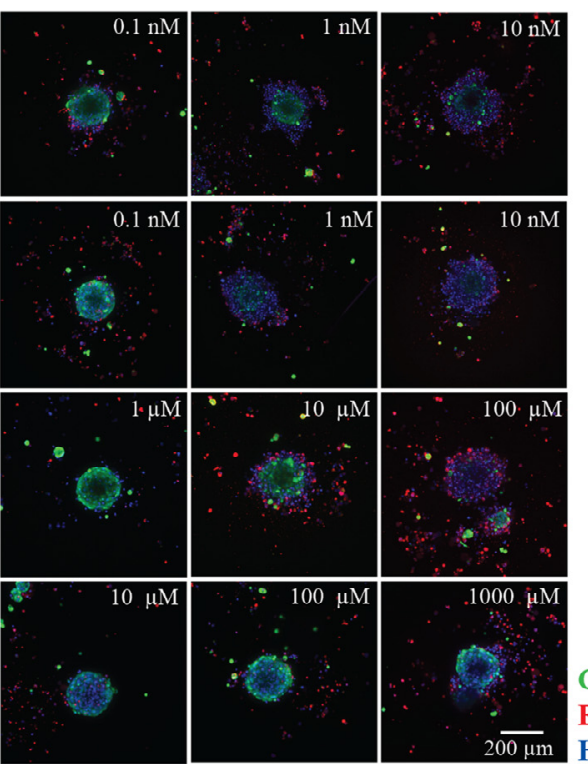

B

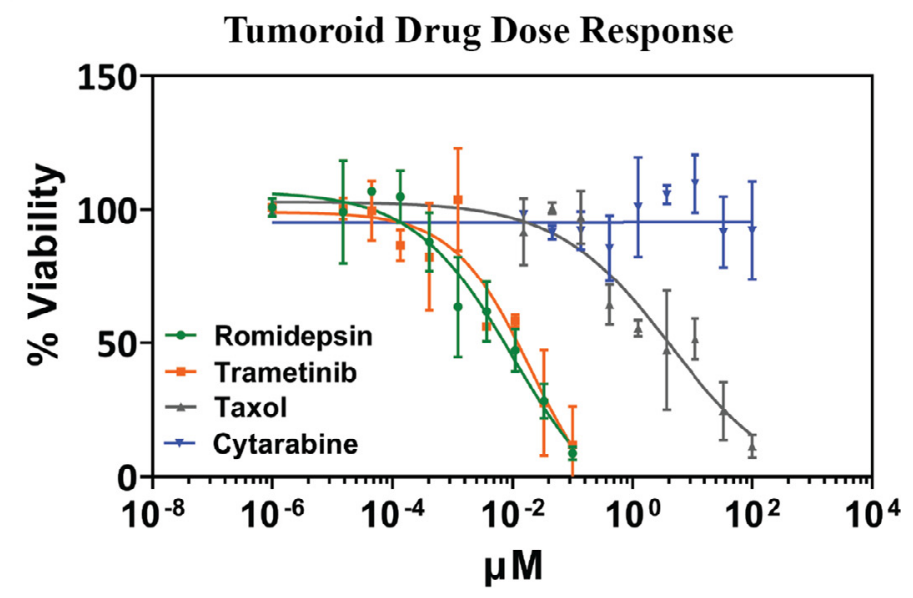

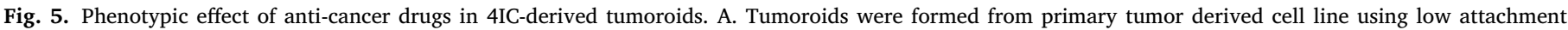

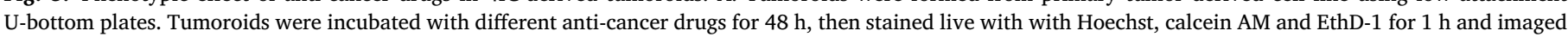

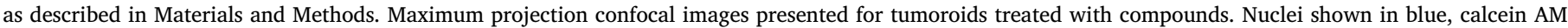

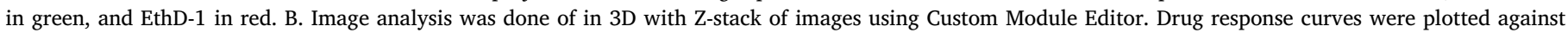
percentage viability as shown for the different drug treatments. Error bars = +/- 1 SD.

Table 1

Comparison of average $\mathrm{IC}_{50}$ values for drug treatment of tumoroids determined by viability staining and analysis of live tumoroids. Viability was determined as $\%$ of live cells.

\begin{tabular}{ll}
\hline Compound & Viability IC $_{50}$ Values \\
\hline Romidepsin & $17.5 \mathrm{nM}$ \\
Trametinib & $25.4 \mathrm{nM}$ \\
Paclitaxel & $10.8 \mu \mathrm{M}$ \\
\hline
\end{tabular}

cytarabine demonstrated cytotoxic activity in 4IC tumoroids, however the concentration responses were significantly different: romidepsin and trametinib had much lower $\mathrm{IC}_{50}$ values $(\sim 10 \mathrm{nM})$ in comparison to paclitaxel ( $>1 \mu \mathrm{M})$ or cytarabine (no significant effect). The paclitaxel value was also much higher than published values observed with the standard breast cancer cell line MCF-7 ( $<10 \mathrm{nM}$ ) [19] and consistent with previously published characterization of the 4IC primary tumor as resistant to taxane therapy [13]. Average data from 4 representative experiments are summarized in Table 1.

\section{Tumoroid biomarker response to compounds}

Two biomarkers of tumor phenotypes, E-cadherin and CD44, were also investigated. E-cadherin is a cell adhesion molecule that is expressed in normal breast tissue and is useful as a phenotypic marker in breast cancer. The E-cadherin protein (encoded by the CDH1 gene) is normally expressed in breast epithelial tissue and functions as a critical part of epithelial cell adhesion and epithelial-to-mesenchymal transition (EMT) [19]. Initial loss of CDH1 (and in some cases restoration of CDH1 after seeding in distal tissue sites) has been associated with transition to migratory and metastatic cancer cell behavior [20,21].

Breast cancers contain a heterogeneous population of cells with a small percentage that possess properties similar to those found in stem cells. One of the widely accepted markers of breast cancer stem cells (BCSCs) is the cell surface marker CD44. CD44 expression is highly up-regulated in BCSCs, and has been implicated in tumorigenesis and metastasis [22]. CD44 promotes a variety of functions independently or in cooperation with other cell-surface receptors through activation of varied signaling pathways like Rho GTPases, Ras-MAPK, and PI3K/AKT pathways to regulate cell adhesion, migration, survival, invasion, and epithelial-mesenchymal transition [23].

To determine the effect of treatment on expression level of Ecadherin and CD44, tumoroids were fixed and stained in Pu.MA System flowchips with fluorescently labeled primary antibodies to E-cadherin and CD44 as described in Methods section. Tumoroids were co-stained with Hoechst nuclear stain to identify individual cells for the image analysis. After staining, tumoroids were washed once and then imaged using ImageXpress Micro Confocal System with 10X objective.

Z-stacks of images were acquired at three Excitation/Emission wavelength pairs to visualize expression of the biomarkers: E-cadherin, CD44 and nuclei (Fig. 6). Treated tumoroids showed reduction in E-cadherin expression compared to vehicle controls for romidepsin and trametinib; similar results published previously have also been observed for paclitaxel [13]. The significance of this for treatment and potential drug resistance is being further investigated. In addition, general disintegration of the tumoroids was observed at higher concentrations along with a decrease in number of E-cadherin and CD44 positive cells (Fig. 6). Together these findings demonstrate the capabilities of our novel technology to use immunostaining to visualize response of biomarkers to various drug treatments in the same system with minimal disruption of fragile tumoroids or organoids.

\section{Evaluation of drug responses in PDX organoids}

PDX-O models are considered a more translational oncology model than tumoroids as they incorporate the multiple cell types found in primary tumors. However, they are not as scalable as tumoroids and as such the latter are a more attractive sample for medium to high throughput drug discovery. We have evaluated drug responses in 4IC PDX-O cultures and compared the results to 4IC tumoroids. The tissues were treated and processed similarly to 4IC tumoroids. Two passages of the PDX-O tissue were prepared, and we evaluated the effects of romidepsin, trametinib and paclitaxel using the flowchip system in combination with 


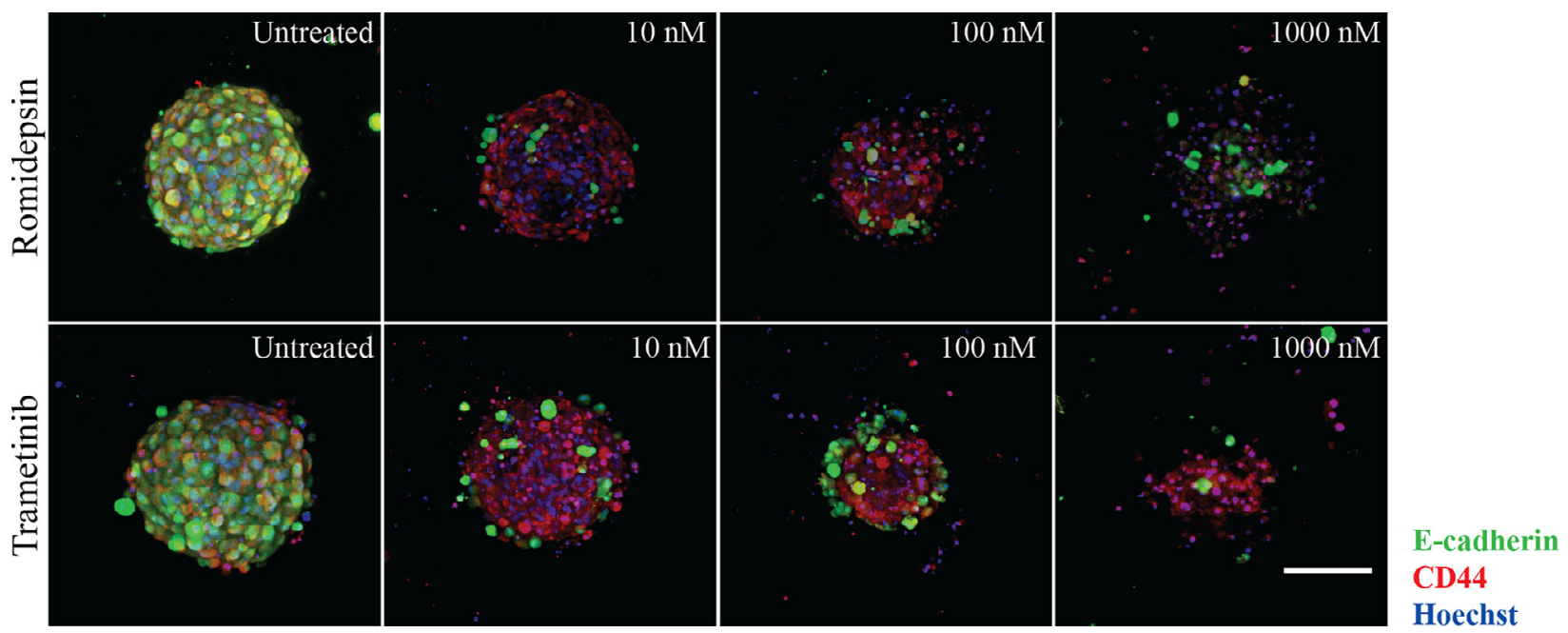

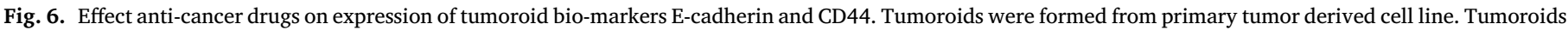

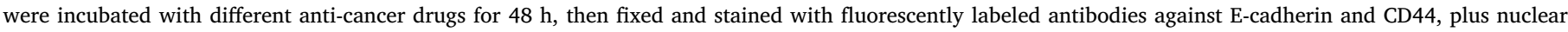

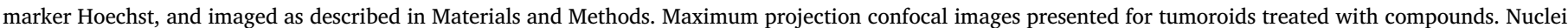
presented in blue, E-cadherin in green, and CD44 in red.

\section{Table 2}

Comparison of response of tumoroids and PDX-Os to different drugs. Responses were similar and shared a common theme of decreased sensitivity to paclitaxel compared to the targeted inhibitors. The tumoroid results presented here were from a different set of experiments than results shown in Table 1 using a different passage of 4IC cells.

\begin{tabular}{llll}
\hline & \multicolumn{2}{l}{ Comparison of $\mathrm{IC}_{50}$ Values $(\mathrm{nM})$ between PDXO and Tumoroids } \\
\cline { 2 - 4 } Cell Type & 4IC Tumoroids & PDXO - 4ICT2 & PDXO - 4ICT6 \\
\hline Romidepsin & $11.3+/-5.8$ & $7.8+/-6.5$ & $21.2+/-4.2$ \\
Trametinib & $4.6+/-0.03$ & $16.8+/-9.6$ & $1.88+/-0.55$ \\
Paclitaxel & $6000+/-2000$ & 10500 & $18000+/-12.6$ \\
\hline
\end{tabular}

high content imaging. The results, presented in Table 2 , were found to be consistent with $4 \mathrm{IC}$ tumoroid response including a high $\mathrm{IC}_{50}$ value for paclitaxel. This is only a first-level comparison of the two models but shows the ability of the Pu-MA System to perform multi-functional profiling with PDX-O tissue.

\section{Metabolic response of tumoroids to treatment}

Energy metabolism of cancer cells has been studied extensively [2426]. As cancer cells multiply, their expansion outgrows angiogenesis so that while they have access to glucose the lack of blood vessels disrupts the level of oxygen needed for the full glucose oxidation. Such a microscopic picture defines one of the fundamental properties of the cancer microenvironment that forces malignant cells to metabolize glucose through the lactic acid cycle. Conversion of glucose to lactic acid, even in the presence of oxygen is known as aerobic glycolysis or the Warburg effect [26]. Elevation of lactate suggests a switch to aerobic glycolysis; tumor cells metabolize glucose into lactate even in the presence of high oxygen [27]. Metabolic alterations in cancer cells are either necessary to cover the energetic demand of the cells or they contribute to the aggressiveness of cancer cells [28]. Recent studies revealed that metabolic alterations of cancer cells play important roles in chemoresistance in breast cancer. A number of studies showed that exposure of cancer cells to chemotherapeutics induces metabolic reprogramming toward increased glycolysis and lactate production $[29,30]$. Therefore, monitoring lactate production over the course of treatment in conjunction with other response endpoints provides valuable information for understanding the dynamics of metabolic perturbations associated with drug response and resistance. It can also be used for the screening of therapies targeting glucose metabolism to overcome resistance to anticancer therapy. Significantly, while there have been many studies using 2D cell models very little data is available for differences in metabolism between 2D and 3D models.

In this study we demonstrated the utility of Pu-MA System to assess the effect of drug treatment on lactate secretion in 4IC tumoroids. The assay was performed in a single tumoroid format and protocol comprised (a) measuring initial tumoroid viability with RT-Glo (b) collection of time-course supernatant samples for the metabolite/lactate analysis and (c) measuring post-treatment tumoroid viability with CTG. A schematic of the complete protocol is shown in Supplementary Fig. 3.

In this assay, as a first step, we incorporated pre-treatment measurement of tumoroid viability using RT-Glo luminescence assay. One of the challenges of working with 3D cell models such as tumoroids is how to normalize the data to the number of cells in each tumoroid. While the starting number of cells can be controlled, there can be variability in cell proliferation and death over $72 \mathrm{~h}$ of tumoroid formation. Another factor to account for is how many tumoroids are loaded into each sample well. We have established best practices of transferring single or multiple tumoroids to the flowchip. However, for the single tumoroid experiments there is a chance of transfer failure leading to no tumoroid in the sample well. Likewise for the experiments using suspension of tumoroids, it is impossible to precisely control the number of spheres (and number of cells in them) transferred which results in natural variability in the number of tumoroids transferred to each sample well. RT-Glo assay uses a non-toxic, cell permeable pro-substrate reagent that is converted to substrate by viable cells and secreted back into the media where it reacts with NanoLuc luciferase to produce light. In the absence of cells, the luminescence signal is extremely low at the background level. The utility of the assay has been demonstrated with 2D cell samples [18] and here we extend its use to 3D cell cultures. As a part of evaluation of RT-Glo usability in 3D setting, we have demonstrated the linear relationship between the cell density/tumoroid size and the RT-Glo luminescence signal (Supplementary Fig. 4). RT-Glo assay provides the means to confirm the successful transfer of tumoroids to the flowchip, to assess the uniformity of the created tumoroids and to normalize the data to the number of viable cells.

4IC tumoroids were dispensed into flowchip sample wells with $20 \mu \mathrm{L}$ of media + RT-Glo reagent. Four connected reagent wells were filled with $20 \mu \mathrm{L}$ of media with vehicle (Untreated) or media + compounds (Treated). Three compounds were tested at three concentrations using a 1:10 serial dilution: Romidepsin $(10 \mathrm{nM}, 1 \mathrm{nM}, 0.1 \mathrm{nM})$, trametinib 
A

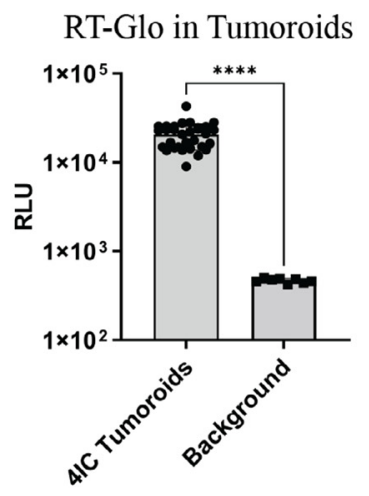

D

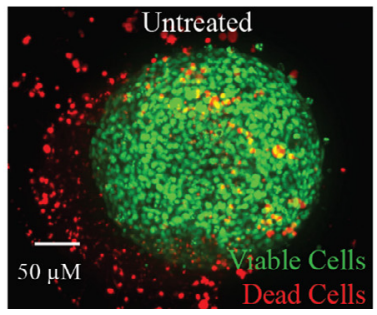

B

Romidepsin

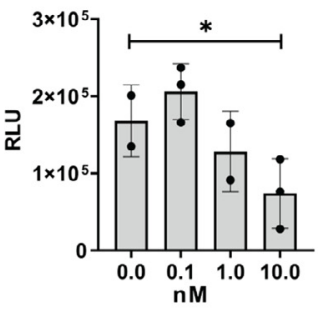

C
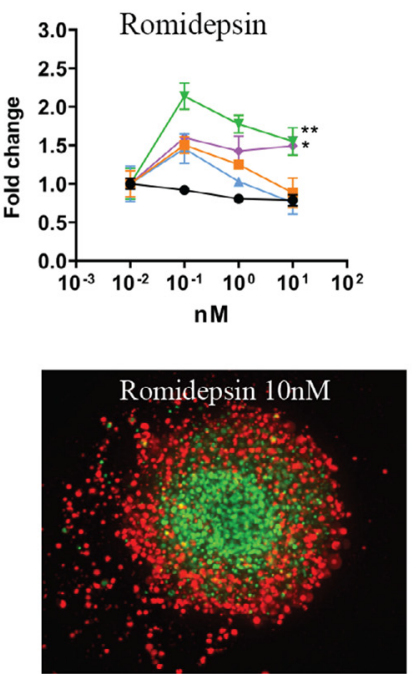

Trametinib
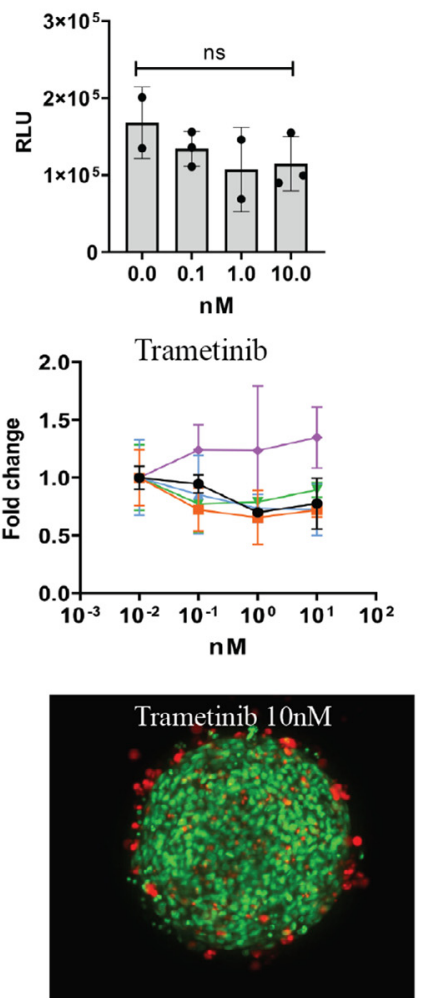

Paclitaxel
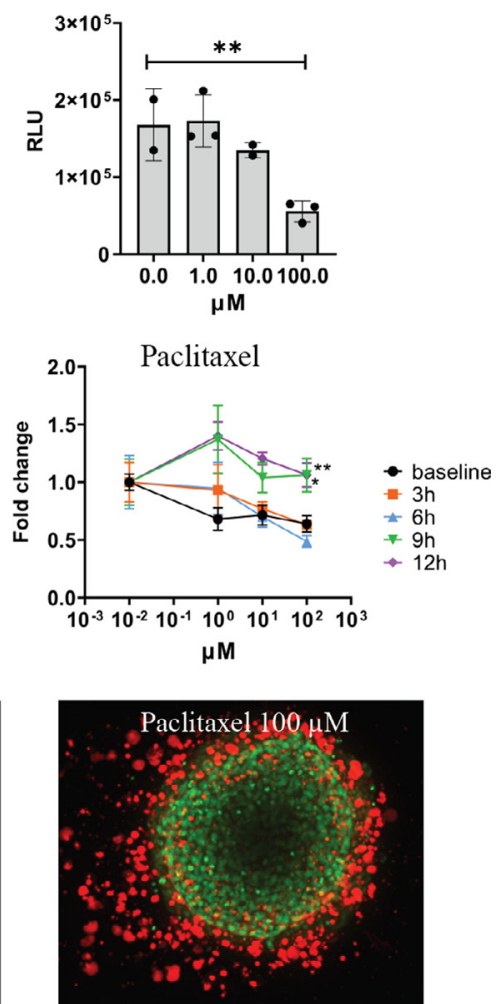

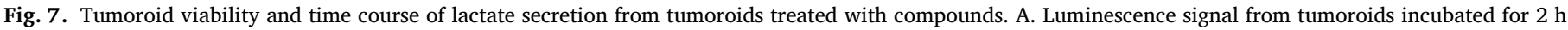

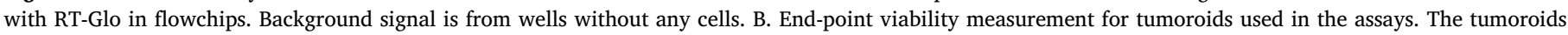

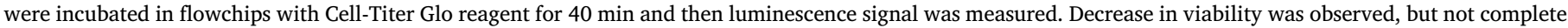

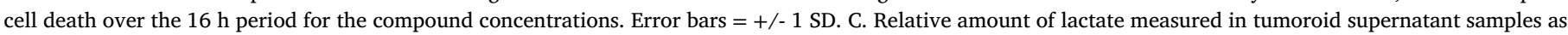

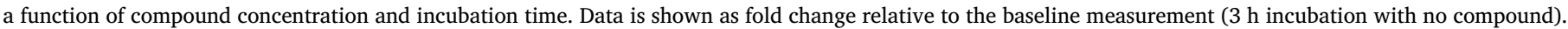

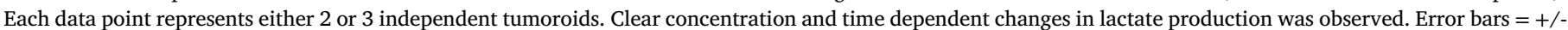

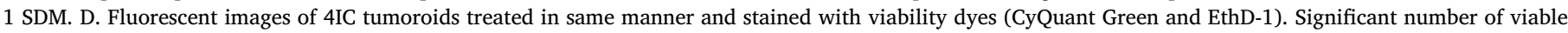

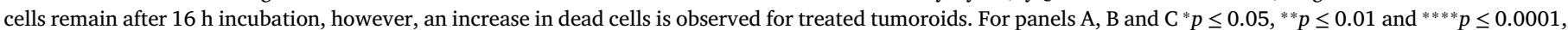
ns: not significant.

(10 nM, $1 \mathrm{nM}, 0.1 \mathrm{nM})$, and paclitaxel $(100 \mu \mathrm{M}, 10 \mu \mathrm{M}, 1 \mu \mathrm{M})$. After RT-Glo measurement was completed, the flowchips were loaded into a $\mathrm{Pu} \cdot \mathrm{MA}$ System in an incubator $\left(37^{\circ} \mathrm{C}, 5 \% \mathrm{CO}_{2}\right)$ and then the treatment and metabolite time-course sampling protocol were performed.

The RT-Glo output prior to treatment is shown in Fig. 7A. Good assay capability was found with a Signal-to-Background of 44 and Signal-toNoise ratio of 690 (Noise $=$ Standard Deviation of Background). The coefficient of variation of signal for the different tumoroid samples was $26 \%$ indicating high starting uniformity in tumoroids size and viability, which is important for the validity of the conclusions.

The supernatant samples were collected at each time point for each drug concentration and for the untreated DMSO vehicle control. Supernatants were analyzed for lactate concentration using the Lactate-Glo assay. 3 independent samples were used for the High and Low concentrations; 2 independent samples were used for the Mid concentrations. Medium background measurement (without cells) was subtracted from all measured luminescence values. All measured values were within the linear range of the 12-point standard curve.

The results for the lactate time course analysis are shown in Fig. 7C. Data is expressed as Fold Change normalized to the untreated DMSO Control for each time point. Two-way ANOVA revealed that there were statistically significant differences in lactate secretion between baseline and measured time points for all three drugs $(F(2.27,19.9)=14.08$ and $p=0.0001$ for Paclitaxel; $\mathrm{F}(2.48,19.2)=13.52$ and $p=0.0001$ for Romidepsin; $\mathrm{F}(1.66,13.26)=10.69$ and $p=0.0025)$, indicating druginduced shift toward glycolysis. Post hoc analysis showed statistically significant increase in lactate secretion over 9 and $12 \mathrm{~h}$ of drug exposure compared to baseline for Paclitaxel ( $p=0.0395$ and 0.0073 , respectively) and for Romidepsin ( $p=0.0096$ and 0.0234 , respectively). Romidepsin demonstrated significant effect of the drug dose on the lactate secretion level $(\mathrm{F}(3,6)=5.324$ and $p=0.0397)$. These drugs belong to different classes of therapeutics (Romidepsin and Trametinib are targeted drugs whereas Paclitaxel is a chemotherapeutic) with different mechanism of action, which could in part explain observed differences. Our observations were in line with previously published reports linking the treatment with paclitaxel with increased lactate production, which plays role in mediating chemoresistance in breast cancer cells [31].

To determine whether reduction in lactate is a function of cell metabolism or reduction in total number of viable cells, tumoroid viability was measured in the flowchip at the end of treatment using the ATP-based CTG luminescence assay . The CTG reagent was added directly to each sample well and incubated for $40 \mathrm{~min}$ at room temperature. The luminescence signal was measured in the flowchip using a plate reader. The results are shown in Fig. 7B. A decrease in cell viability was seen with all three compounds at the highest dose after $15 \mathrm{~h}$ of treatment. However, significant ATP signal was still seen indicating only partial killing of cells and many viable cells remained intact and metabolically active. This is consistent with the imaging viability data response for similar 4IC tumoroids treated with the same compounds (Fig. 7D). Given that cell death is expected to increase over time, the increase in lactate observed at the $15 \mathrm{~h}$ time points may indicate an even higher increase in glycolysis. There can be multiple dynamics involved 
including the dissociation of the tumoroid extracellular bonds leading to better penetration of the assay reagents to the core of the tumoroid or increase in metabolism of the inner tumoroid cells as they have better access to oxygen and media nutrients. Additional experiments are required to understand these interacting factors.

\section{Discussion}

3D cell models such as tumoroids and organoids have transformed pre-clinical drug treatment studies and have become a powerful tool to better model diseases and understand response of tissue to treatment. We introduce here a novel flowchip based system for automating tumoroid assay steps that allows multifunctional profiling of single tumor tissue samples to provide superior insight into cellular response to therapeutic compounds. A key aspect of the Pu.MA System is its compatibility with high content imaging and analysis. High resolution confocal imaging of multiple cellular components allows assessment of viability, biomarker expression, and other phenotypic readouts in three dimensions. This is important for tumor models as it is known that tumors can have hypoxic cores that respond differently to treatments [32]. The ease-of-use of the flowchip plate design, conforming to the SLAS/ANSI standard for 384 multiwell plates, makes acquiring images with automated imaging platforms straightforward and opens up the possibility to acquire multiple image time points during an assay. Another key feature of the Pu-MA System is multiple automated media exchanges that occur without loss of or disruption to the sample. This allows timecourse sampling of tumoroid supernatants and enables characterization of metabolism dynamics. The system is compatible with other 3D cell models including spheroids, Matrigel-grown organoids, and other tissue samples making it versatile for multiple assay types.

We demonstrated utility of the Pu.MA System with an important 3D cell model: patient-derived tumoroids. These tissue samples represent the genomic profile of the original patient tumor and can recapitulate the patient response to therapy $[12,13]$. The $\mathrm{IC}_{50}$ value measured from the paclitaxel concentration response of the tumoroids was found to be 100 times higher than the two other compounds. In fact, this result was different with published $\mathrm{IC}_{50}$ values for paclitaxel in assays that used same cancer cell line, but in 2D assay format $(\sim 20 \mathrm{nM})$ [13]. In contrast, the response of 4IC tumoroids to paclitaxel is consistent with the lack of response of patient to paclitaxel treatment suggesting utility of our model system [13].

However, since the only one tumor-derived cell line was used in the study, this limits the interpretation of the data in context of the tumor type and applications to clinical translatability. Paclitaxel has been shown previously to effect cancer cell spheroids such as HCT116 and other tumor cell lines [16]. There are also studies of the breast cancer cell line MCF-7 response to paclitaxel [19]. Comparison to standard cell lines and other patient-derived tumor lines using the same assays would be required to definitively characterize the 4IC response and clinical translatability of the results. While this work was focusing on the method development, more extensive research of this particular tumor type, including multiple samples, will be necessary for understanding biology and finding new compounds for patient treatment.

Interestingly, the E-cadherin expression decreased at the lowest concentrations of all treatments studied and might provide insight into the ECM response of TNBC tumors. Loss of E-cadherin has been observed in many types of cancer and is associated with epithelial-to-mesenchymal transition, cell migration and invasion [33,34]. Important differences have been observed in behavior of E-cadherin in 2D and 3D cell models [2]. There is also substantial amount of data showing strong correlation between E-cadherin expression and the ability of cells to form 3D spheres in vitro. Silencing of E-cadherin in breast cancer cells prevented the formation of mammospheres, whereas overexpression of E-cadherin in non-spherogenic cells resulted in sphere formation. This observation links the process of mammopshere formation with stem like features in breast cancer cells [35]. These data show that treatment of adherent
PDX-derived cells in adherent, or 2D culture conditions for some anticancer agents tested in vitro does not translate to treating the matched intact patient-derived tumoroids. Such complex biology of E-cadherin should be taken into account when interpreting results obtained from $3 \mathrm{D}$ in vitro models. The observed reduction in E-cadherin expression in our study due to compound treatment warrants further investigation.

Finally, the characterization of lactate secretion and its implications for dynamic metabolism response to treatment is important. It is accepted that tumors undergo aerobic glycolysis, but the exact role of that in tumor resistance and whether it can be used as a therapeutic target is not known. However, the ability to dynamically monitor changes in glycolysis opens up many experimental possibilities.

There are a few limitations of the current system such as sample throughput, size of tissue samples, and ability to use with long-term culture. Some of these limitations are currently being addressed. In addition, the single timepoint used in the current study limits the ability to draw conclusions about relative drug sensitivities as some compounds may require longer times to effect cell fate. However, the ability to perform multifunctional profiling of complex tumoroid samples provides a high value to researchers trying to understand disease mechanism and resistance to therapies. The use of time-course sampling of conditioned media allows interrogation of the dynamics of tumoroid metabolism in response to treatment. This is critical because tumoroid metabolism can change on a much faster time scale than viability so using a single "cell-death" endpoint does not provide any insight into the dynamics of that death. The system is extendable beyond oncology applications and will be useful for liver toxicology, functional cardio-assays, intestinal inflammation, and other important areas. Ultimately, flowchip automation with protected 3D cell model samples coupled with multiplexed downstream analysis by employing high-content imaging and sensitive luminescence assays is a powerful approach for drug discovery and disease modeling.

\section{Conclusion}

We demonstrated here a method suitable for automated processing of tumoroid samples and phenotypic analysis of compound effects using multi-parametric read-outs, combining imaging and metabolic analysis methods. These methods provide an innovative strategy to improve drug sensitivity studies of individual tumor types to specific drug classes, with important implications for the future development of personalized medicine, prospective prediction of drug responses and selection of best drug combination for treatment of specific patients.

\section{Declaration of Competing Interest}

The authors declared the following potential competing interests with respect to the research, authorship, and/or publication of this article: The authors EFC and EN are employed by Protein Fluidics, which manufactures the PuMA System. The authors OS and MH are employed by Molecular Devices, which manufactures the ImageXpress Micro Confocal system. The authors CKB, MDM, MSA, BMC-B, and MEB declare no competing interests.

This article is being reproduced in print post-publication in a sponsored print collection for distribution. The company sponsoring the print collection was not involved in the editorial selection or review of this article.

\section{Acknowledgments}

This work was supported by grants from National Institute of Health (NIH) (Grants No. R01-CA174785-01A1 and R01CA125806-02). We acknowledge and thank Krewe de Pink for their financial support of this project and dedication to support breast cancer patients in the local New Orleans community. We also would like to thank Promega for providing 
a GloMax plate reader and RT-Glo, CTG, and Lactate-Glo assay kits, and Greiner Bio-One for supplying NanoShuttle reagent

\section{Supplementary materials}

Supplementary material associated with this article can be found, in the online version, at doi:10.1016/j.slasd.2022.01.006.

\section{References}

[1] Matossian MD, Giardina AA, Wright MK, et al. Patient-derived xenografts as an innovative surrogate tumor model for the investigation of health disparities in triple negative breast cancer. Womens Health Rep 2020;1:383-92 (New Rochelle).

[2] Matossian MD, Burks HE, Elliott S, et al. Drug resistance profiling of a new triple negative breast cancer patient-derived xenograft model. BMC Cancer 2019;19:205.

[3] Toniatti C, Jones P, Graham H, et al. Oncology drug discovery: planning a turnaround. Cancer Discov 2014;4:397-404.

[4] Gupta N, Liu JR, Patel B, et al. Microfluidics-based 3D cell culture models: utility in novel drug discovery and delivery research. Bioeng Transl Med 2016;1:63-81.

[5] Yin X, Mead BE, Safaee H, et al. Engineering stem cell organoids. Cell Stem Cell 2016;18:25-38.

[6] Passier R, Orlova V, Mummery C. Complex tissue and disease modeling using hiPSCs. Cell Stem Cell 2016;18:309-21.

[7] Park SE, Georgescu A, Huh D. Organoids-on-a-chip. Science 2019;364:960-5.

[8] Wardwell-Swanson J, Suzuki M, Dowell KG, et al. A framework for optimizing high-content imaging of 3D models for drug discovery. SLAS Discov 2020;25:709-22.

[9] Schutgens F, Clevers H. Human organoids: tools for understanding biology and treating diseases. Annu Rev Pathol Mech Dis 2020;15:211-34.

[10] Frappart PO, Walter K, Gout J, et al. Pancreatic cancer-derived organoids - a disease modeling tool to predict drug response. United Eur Gastroenterol J 2020;8:594-606.

[11] Cromwell EF, Leung M, Hammer M, et al. Disease modeling with 3D cell-based assays using a novel flowchip system and high-content imaging. SLAS Technol 2021;26:237-48.

[12] Matossian MD, Elliott S, Van Hoang T, et al. NEK5 activity regulates the mesenchymal and migratory phenotype in breast cancer cells. Breast Cancer Res Treat 2021.

[13] Matossian MD, Chang T, Wright MK, et al. In-depth characterization of a new patient-derived xenograft model for metaplastic breast carcinoma to identify viable biologic targets and patterns of matrix evolution within rare tumor types. Clin Transl Oncol 2021.

[14] Chang TC, Matossian MD, Elliott S, et al. Evaluation of deacetylase inhibition in metaplastic breast carcinoma using multiple derivations of preclinical models of a new patient-derived tumor. PLoS One 2020;15:e0226464.

[15] Matossian MD, Burks HE, Bowles AC, et al. A novel patient-derived xenograft model for claudin-low triple-negative breast cancer. Breast Cancer Res Treat 2018;169:381-90.
[16] Sirenko O, Mitlo T, Hesley J, et al. High-content assays for characterizing the viability and morphology of 3D cancer spheroid cultures. Assay Drug Dev Technol 2015;13:402-14.

[17] Grimm FA, Iwata Y, Sirenko O, et al. High-content assay multiplexing for toxicity screening in induced pluripotent stem cell-derived cardiomyocytes and hepatocytes. Assay Drug Dev Technol 2015;13:529-46.

[18] Duellman SJ, Zhou W, Meisenheimer P, et al. Bioluminescent, nonlytic, realtime cell viability assay and use in inhibitor screening. Assay Drug Dev Technol 2015;13:456-65.

[19] Liebmann JE, Cook JA, Lipschultz C, et al. Cytotoxic studies of paclitaxel (Taxol) in human tumour cell lines. Br J Cancer 1993;68:1104-9.

[20] Singhai R, Patil VW, Jaiswal SR, et al. E-cadherin as a diagnostic biomarker in breast cancer. N Am J Med Sci 2011;3:227-33.

[21] Corso G, Figueiredo J, De Angelis SP, et al. E-cadherin deregulation in breast cancer. J Cell Mol Med 2020;24:5930-6.

[22] Smith SM, Cai L. Cell specific CD44 expression in breast cancer requires the interaction of AP-1 and NFkappaB with a novel cis-element. PLoS One 2012;7:e50867.

[23] Al-Othman N, Alhendi A, Ihbaisha M, et al. Role of CD44 in breast cancer. Breast Dis 2020;39:1-13.

[24] Gosalvez M, Garcia-Suarez S, Lopez-Alarcon L. Metabolic control of glycolysis in normal and tumor permeabilized cells. Cancer Res 1978;38:142-8.

[25] Warburg O. On the origin of cancer cells. Science 1956;123:309-14.

[26] Devic S. Warburg effect - a consequence or the cause of carcinogenesis? J Cancer 2016;7:817-22.

[27] Yang J, Jin X, Yan Y, et al. Inhibiting histone deacetylases suppresses glucose metabolism and hepatocellular carcinoma growth by restoring FBP1 expression. Sci Rep 2017;7:43864.

[28] Braun LM, Lagies S, Guenzle J, et al. Metabolic adaptation during nab-paclitaxel resistance in pancreatic cancer cell lines. Cells 2020:9.

[29] Dong Q, Zhou C, Ren H, et al. Lactate-induced MRP1 expression contributes to metabolism-based etoposide resistance in non-small cell lung cancer cells. Cell Commun Signal 2020;18:167.

[30] Barnes EME, Xu Y, Benito A, et al. Lactic acidosis induces resistance to the pan-Akt inhibitor uprosertib in colon cancer cells. Br J Cancer 2020;122:1298-308.

[31] Hou L, Zhao Y, Song GQ, et al. Interfering cellular lactate homeostasis overcomes Taxol resistance of breast cancer cells through the microRNA-124-mediated lactate transporter (MCT1) inhibition. Cancer Cell Int 2019;19:193.

[32] Muz B, de la Puente P, Azab F, et al. The role of hypoxia in cancer progression, angiogenesis, metastasis, and resistance to therapy. Hypoxia 2015;3:83-92 (Auckl).

[33] Horne HN, Oh H, Sherman ME, et al. E-cadherin breast tumor expression, risk factors and survival: pooled analysis of 5933 cases from 12 studies in the breast cancer association consortium. Sci Rep 2018;8:6574.

[34] Liu J, Sun X, Qin S, et al. CDH1 promoter methylation correlates with decreased gene expression and poor prognosis in patients with breast cancer. Oncol Lett 2016;11:2635-43.

[35] Manuel Iglesias J, Beloqui I, Garcia-Garcia F, et al. Mammosphere formation in breast carcinoma cell lines depends upon expression of E-cadherin. PLoS One 2013;8:e77281 